\title{
Discovery of a Potential Liver Fibrosis Inhibitor from a Mushroom Endophytic Fungus by Genome Mining of a Silent Biosynthetic Gene Cluster
}

Jin-Tao Cheng ${ }^{\mathrm{a}, \mathrm{g} \sharp}$, Han-Min Wang ${ }^{\mathrm{b}, \mathrm{h} \#}$, Jia-Hui Yu ${ }^{\mathrm{d}}$, Chen-Fan Sun ${ }^{\mathrm{a}, \mathrm{g}}$, Fei Cao ${ }^{\mathrm{a}, \mathrm{g}}$, Xin-Hang Jiang , $^{\mathrm{e}}$ Xin-Ai Chen", , Qing-Wei Zhao ${ }^{\mathrm{a}}$, Li-She Gan ${ }^{\mathrm{d}, \mathrm{f}}$, Rong-Rong Xie ${ }^{\mathrm{b}}$, Shi-Lei Wang ${ }^{\mathrm{i}}$, Jia Li ${ }^{\mathrm{b}, \mathrm{h}}$, Yi Zang ${ }^{\mathrm{b}, \mathrm{h} *}$, Xu-Ming Mao $\mathrm{Ma}^{\mathrm{a}, \mathrm{c}, \mathrm{g} *}$

${ }^{a}$ Institute of Pharmaceutical Biotechnology \& Research Center for Clinical Pharmacy, The First Affiliated Hospital, School of Medicine, Zhejiang University, Hangzhou 310058, China

${ }^{b}$ National Center for Drug Screening, State Key Laboratory of Drug Research, Shanghai Institute of Materia Medica, Chinese Academy of Sciences, Shanghai, 201203, China

${ }^{\mathrm{c}}$ State Key Laboratory of Bioactive Substance and Function of Natural Medicines, Institute of Materia Medica, Chinese Academy of Medical Sciences and Peking Union Medical College, Beijing 100050, P. R. China

${ }^{\mathrm{d} C o l l e g e ~ o f ~ P h a r m a c e u t i c a l ~ S c i e n c e s, ~ Z h e j i a n g ~ U n i v e r s i t y, ~ H a n g z h o u ~ 310058, ~ C h i n a ~}$

${ }^{\mathrm{e}}$ Equipment and Technology Service Platform, College of Life Sciences, Zhejiang University, Hangzhou 310058, China

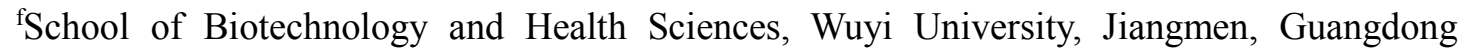
529020, People's Republic of China

gZhejiang Provincial Key Laboratory for Microbial Biochemistry and Metabolic Engineering, Hangzhou 310058, China

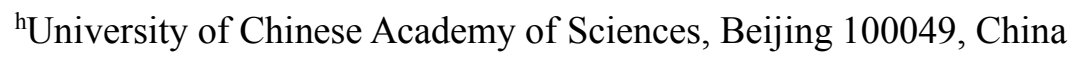

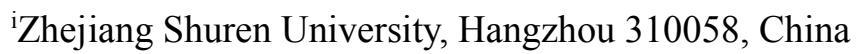

\#These authors contributed equally to this work.

*To whom correspondence should be addressed:

Yi Zang, E-mail: yzang@simm.ac.cn; Tel: 86-021-50801313.

Xu-Ming Mao, E-mail: xmmao@zju.edu.cn; Tel: 86-571-88981335. 


\section{Content}

Supplementary Tables: $\quad$ Page

Table S1. Strains and plasmids used in this work $\quad$ S3

Table S2. Oligonucleotides used in this study $\quad$ S4

Table S3. Primers used in RT-PCR $\quad$ S5

Table S4. Quantitative real-time PCR with the sequence-specific primer pairs S6

$\begin{array}{ll}\text { Table S5. Detailed annotation of each gene in gene cluster } \text { mcal7 } & \text { S7 }\end{array}$

Table S6. NMR data of compound $1 \quad$ S8

Table S7. Energy (298.15 K) analysis for (3S)-1 $\quad$ S9

Table S8. Calculated ECD data for (3S)-1 in solvent phase $\quad$ S10

Table S9. Calculated specific OR data for (3S)-1 $\quad$ S13

Supplementary Figures:

Figure S1. Determination of the structure of compound $1 \quad$ S14

Figure S2. ${ }^{1} \mathrm{H}$ NMR spectrum of $\mathbf{1}$ in DMSO $(500 \mathrm{MHz}) \quad \mathrm{S} 15$

Figure S3. ${ }^{13} \mathrm{C}$ NMR spectrum of $\mathbf{1}$ in DMSO $\quad$ S16

$\begin{array}{ll}\text { Figure S4. DEPT } 90 \text { NMR spectrum of } \mathbf{1} \text { in DMSO } & \text { S17 }\end{array}$

Figure S5. DEPT 135 NMR spectrum of 1 in DMSO $\quad$ S18

$\begin{array}{lr}\text { Figure S6. The HSQC spectrum of } \mathbf{1} \text { in DMSO } & \text { S19 }\end{array}$

$\begin{array}{ll}\text { Figure S7. The HMBC spectrum of } \mathbf{1} \text { in DMSO } & \text { S20 }\end{array}$

$\begin{array}{ll}\text { Figure S8. Two candidate absolute configurations of } 1 & \text { S21 }\end{array}$

Figure S9. B3LYP/6-31G(d) optimized lowest energy conformer for (3S)-1 $\quad$ S22

$\begin{array}{ll}\text { Figure S10. Multiple alignment of DKC domain sequences } & \text { S23 }\end{array}$

$\begin{array}{lr}\text { Reference } & \text { S24 }\end{array}$ 
Table S1. Strains and plasmids used in this work

\begin{tabular}{lll}
\hline Strains/Plasmid & Description & Source \\
\hline C. arbuscula $\triangle$ aurA & $\begin{array}{l}\text { deficient of AurA (encoding aurovertin polyketide } \\
\text { synthase) }\end{array}$ & 1 \\
Plasmids & $\begin{array}{l}\text { E. coli-A. tumefaciens shuttle expression vector, } \\
\text { kanamycin } \\
\text { pFGL815N }\end{array}$ & 1 \\
pFGL-teflp & $\begin{array}{l}\text { pFGL-neoR derived, carrying teflp } \\
\text { pFGL-teflp derived, carrying TF17 at BamHI }\end{array}$ & 1 \\
pFGL-teflp-TF17 & pFGL-teflp derived, carrying PKS17 at BamHI & This study \\
pFGL-teflp-PKS17 & &
\end{tabular}


Table S2. Oligonucleotides used in this study

\begin{tabular}{ll}
\hline Primer & Sequence \\
\hline TF17-F & CTTATCGTCGTCATCCTTGTAATCTATAATGCAAAGGCTCTCTTTAAGTCG \\
TF17-R & CACTCACAACCGCAAAAGGATCCATGGACCCGACTACTGAGGAAG \\
PKS17-1-F & CGTCGTCATCCTTGTAATCGGATCCTCATCTTCGCGATCTAAGCCTAGC \\
PKS17-1-R & GCGACTCTTTGAACTAGCCAGAC \\
PKS17-2-F & CCACAGGAGCTGTTTTTGGTCC \\
PKS17-2-R & CAAACACTCACAACCGCAAAAATGACGAAAACGACATCGTCGTC \\
\hline
\end{tabular}


Table S3. Primers used in RT-PCR.

\begin{tabular}{|c|c|}
\hline Primer & Sequence \\
\hline A5154-RT-F & CAACCCCAGCCCACTCTTC \\
\hline A5154-RT-R & GCCTACACCACCTACCGG \\
\hline A5155-RT-F & CTTGCTGGAGATTCTGCGC \\
\hline A5155-RT-R & CAGAAGCAGCATCACGCAG \\
\hline A5156-RT-F & CTCAATGGGACGGATCCCG \\
\hline A5156-RT-R & CCGATGAGGATACGCGATG \\
\hline A5157-RT-F & GTGGATCGACACCAGGGG \\
\hline A5157-RT-R & CTGGGAAGTGGATCGGCTATC \\
\hline A5158-RT-F & CTGGCGCAGAGGATGTGC \\
\hline A5158-RT-R & GCCGAGCTTTACGGCTGG \\
\hline A5159-RT-F & GGCTTCACCAGAACTCCATGG \\
\hline A5159-RT-R & GTTGCGTGAAGAGGTGGCC \\
\hline A5160-RT-F & GTAGGCGGCCGTTTGATC \\
\hline A5160-RT-R & CCAAGCTTGCAGGCGGAAG \\
\hline A5161-RT-F & CTAGGGAGGTCAGGCCACAC \\
\hline A5161-RT-R & GGACAATGTCTTCAATCTGCCC \\
\hline A5162-RT-F & GAATCGACGAACCACCTCGC \\
\hline A5162-RT-R & CGTCATCTTGTTCGATGCGTTG \\
\hline A5163-RT-F & GCTTCCGACTGTTGCCTC \\
\hline A5163-RT-R & GTACAGAGATGGCCGTTACC \\
\hline A5164-RT-F & GATGCTCGCTGTAGCGGC \\
\hline A5164-RT-R & GCAACACTGTGCCGTTGAG \\
\hline A5165-RT-F & GGCTCCGCTACAAGCATTG \\
\hline A5165-RT-R & GACACCAAAGACTTGCAGCG \\
\hline A5166-RT-F & GGCGCAACGTCTTTGACC \\
\hline A5166-RT-R & GCTGGTATTACCCGGTCCG \\
\hline A5167-RT-F & GGCTCCСТCTCTGAACTCTC \\
\hline A5167-RT-R & CATATGGTTCGCCCTGAAGG \\
\hline A5168-RT-F & CCGCAAGTCTGAGTAGCG \\
\hline A5168-RT-R & GATGAGGTCCACTGCATTGG \\
\hline A5169-RT-F & GCGTACATCCACCTTTCCAC \\
\hline A5169-RT-R & CGGCGTATTGGTAGCCTAG \\
\hline actA-F & GCGTCACCCACCAGAGAGG \\
\hline $\operatorname{act} A-R$ & GTAGCGGCGAGCGCGAACAC \\
\hline tubC-F & CCAACATCTTGCGGGTGCG \\
\hline tubC-R & GCAAACCGGTACATTGCAC \\
\hline gpdA-F & CATCGTCTTCCGCAATG \\
\hline gpdA-R & CTGCCGTCGTAGGTCTTC \\
\hline
\end{tabular}


Table S4. Quantitative real-time PCR with the sequence-specific primer pairs listed.

\begin{tabular}{lll}
\hline Genes & Sense Primer (5'-3') & AntiSense Primer (5’'3’) \\
\hline Human GAPDH & AAGAAGGTGGTGAAGCAGG & AGGTGGAGGAGTGGGTGTCG \\
Human $\alpha$-SMA & ACTGGGACGACATGGAAAAG & TAGATGGGGACATTGTGGGT \\
Human collagen1 $\alpha 1$ & AGTCTGTCCTGCGTCCTCTG & TGTTTGGGTCATTTCCACAT \\
\hline
\end{tabular}


Table S5. Detailed annotation of each gene in gene cluster $\mathrm{mcal} 17$

\begin{tabular}{|c|c|c|c|}
\hline Gene & $\begin{array}{l}\text { Length } \\
\text { (bp) }\end{array}$ & Predicted function & Host with homologous proteins \\
\hline A05155 & 1235 & Putative transcriptional regulator LovE & Madurella mycetomatis \\
\hline A05156 & 1467 & $O$-methyltransferase & Cryphonectria parasitica $\mathrm{EP} 155$ \\
\hline A05157 & 1835 & MFS gliotoxin efflux transporter GliA & Dissoconium aciculare CBS 342.82 \\
\hline A05158 & 1899 & FAD binding domain-containing protein & Cryphonectria parasitica $\mathrm{EP} 155$ \\
\hline A05159 & 2001 & Cytochrome P450 monooxygenase GliC2 & Cryphonectria parasitica $\mathrm{EP} 155$ \\
\hline A05160 & 1780 & 6-hydroxy-D-nicotine oxidase & Madurella mycetomatis \\
\hline A05161 & 1035 & 1-aminocyclopropane-1-carboxylate oxidase & Madurella mycetomatis \\
\hline A05162 & 1554 & Monooxygenase, $\mathrm{FAD}$ binding domain $\mathrm{CpaO}$ & Curvularia pallescens \\
\hline A05163 & 12298 & Polyketide synthase-nonribosomal peptide synthetase & Madurella mycetomatis \\
\hline A05164 & 1577 & Hypothetical protein G6M90_00g105960 & Metarhizium brunneum \\
\hline A05165 & 1101 & Aflatoxin B1 aldehyde reductase member 3 & Trichoderma harzianum \\
\hline A05166 & 1523 & Protein related to amidohydrolase & Purpureocillium lilacinum \\
\hline A05167 & 3785 & Hypothetical protein CDD83_359 & Cordyceps sp. RAO-2017 \\
\hline A05168 & 1879 & Hypothetical protein M431DRAFT_123853 & Trichoderma harzianum \\
\hline
\end{tabular}


Table S6. NMR data of compound 1

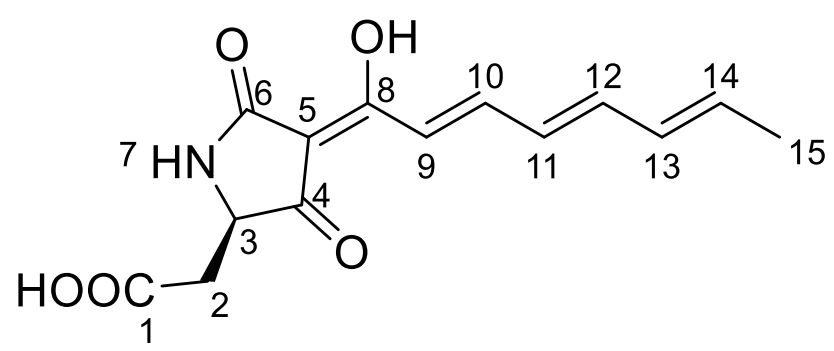

\begin{tabular}{|c|c|c|c|}
\hline & $\delta_{\mathrm{H}}(J$ in $\mathrm{Hz})$ & $\delta_{\mathrm{C}}$ & HMBC \\
\hline 1 & & 171.2 & \\
\hline 2 & $\begin{array}{l}2.58(1 \mathrm{H}, \mathrm{dd}, J=6.2 \\
16.8) \\
2.63(1 \mathrm{H}, \mathrm{dd}, J=4.5 \\
16.8)\end{array}$ & 35.7 & $1,3,4$ \\
\hline 3 & $4.06(1 \mathrm{H}, \mathrm{s})$ & 58.1 & 1,6 \\
\hline 4 & & 194.5 & \\
\hline 5 & & 100.3 & \\
\hline 6 & & 174.6 & \\
\hline 7 & $8.82(1 \mathrm{H}, \mathrm{s})$ & & $3,4,5$ \\
\hline 8 & & 172.3 & \\
\hline 9 & $7.00(1 \mathrm{H}, \mathrm{d}, J=15.3)$ & 119.8 & 8,11 \\
\hline 10 & $\begin{array}{l}7.46(1 \mathrm{H}, \mathrm{dd}, J=15.2, \\
11.5)\end{array}$ & 144.3 & 8,12 \\
\hline 11 & $\begin{array}{l}6.47(1 \mathrm{H}, \mathrm{dd}, J=14.7 \text {, } \\
11.6)\end{array}$ & 128.7 & $9,10,13$ \\
\hline 12 & $\begin{array}{l}6.84(1 \mathrm{H}, \mathrm{dd}, J=14.8, \\
10.9)\end{array}$ & 144.0 & $10,13,14$ \\
\hline 13 & $6.29(1 \mathrm{H}, \mathrm{m})$ & 131.7 & $11,12,15$ \\
\hline 14 & $\begin{array}{l}6.08(1 \mathrm{H}, \mathrm{dt}, J=15.0 \\
7.0)\end{array}$ & 137.2 & 12,15 \\
\hline 15 & $1.81(3 \mathrm{H}, \mathrm{d}, J=6.8)$ & 18.6 & $12,13,14$ \\
\hline
\end{tabular}


Table S7. Energy (298.15 K) analysis for (3S)-1

\begin{tabular}{|l|l|l|l|}
\hline Conf. & $G($ Hartree $)$ & $\Delta G(\mathrm{kcal} / \mathrm{mol})$ & Boltzmann Distribution \\
\hline C1 & -973.169798 & 0 & 1 \\
\hline
\end{tabular}


Table S8. Calculated ECD data for (3S)-1 in solvent phase

\begin{tabular}{|c|c|c|}
\hline \multirow[b]{2}{*}{ State } & \multicolumn{2}{|l|}{$\mathrm{C} 1$} \\
\hline & $\begin{array}{l}\text { Excitation } \\
\text { energies }(e V)\end{array}$ & Rotatory Strengths* \\
\hline 1 & 3.5046 & 4.9944 \\
\hline 2 & 3.9878 & 0.0834 \\
\hline 3 & 4.6552 & 4.0184 \\
\hline 4 & 5.2803 & 1.628 \\
\hline 5 & 5.4706 & -11.5693 \\
\hline 6 & 5.5137 & -1.8047 \\
\hline 7 & 5.5843 & 2.934 \\
\hline 8 & 5.6945 & 1.3285 \\
\hline 9 & 5.7953 & -1.549 \\
\hline 10 & 6.0966 & -4.3043 \\
\hline 11 & 6.4226 & 3.4586 \\
\hline 12 & 6.4911 & -0.4105 \\
\hline 13 & 6.6734 & 31.3064 \\
\hline 14 & 6.7323 & -30.8999 \\
\hline 15 & 6.805 & 3.9161 \\
\hline 16 & 7.041 & 0.7703 \\
\hline 17 & 7.0835 & 4.1226 \\
\hline 18 & 7.1438 & 3.7963 \\
\hline 19 & 7.2124 & -5.8814 \\
\hline 20 & 7.2546 & -1.0719 \\
\hline 21 & 7.3036 & -0.3697 \\
\hline 22 & 7.3858 & -1.3424 \\
\hline
\end{tabular}




\begin{tabular}{|c|c|c|}
\hline 23 & 7.4797 & 7.7377 \\
\hline 24 & 7.5276 & -1.4937 \\
\hline 25 & 7.5489 & 3.4946 \\
\hline 26 & 7.6263 & 3.8767 \\
\hline 27 & 7.7113 & 0.477 \\
\hline 28 & 7.7564 & 5.3893 \\
\hline 29 & 7.7866 & -8.5243 \\
\hline 30 & 7.8373 & -21.1901 \\
\hline 31 & 7.9461 & 8.1571 \\
\hline 32 & 8.0035 & 16.304 \\
\hline 33 & 8.0621 & -8.7079 \\
\hline 34 & 8.0783 & 1.8153 \\
\hline 35 & 8.1601 & -15.9459 \\
\hline 36 & 8.2365 & 2.841 \\
\hline 37 & 8.2434 & -7.2175 \\
\hline 38 & 8.294 & 8.6023 \\
\hline 39 & 8.3377 & 10.6153 \\
\hline 40 & 8.445 & -24.5223 \\
\hline 41 & 8.4576 & 26.6996 \\
\hline 42 & 8.4759 & -2.3336 \\
\hline 43 & 8.5349 & 83.9509 \\
\hline 44 & 8.6067 & -31.1818 \\
\hline 45 & 8.6624 & -8.8032 \\
\hline 46 & 8.6978 & -35.5941 \\
\hline 47 & 8.7121 & 11.5778 \\
\hline 48 & 8.7238 & -9.1289 \\
\hline
\end{tabular}




\begin{tabular}{|l|l|l|}
\hline 49 & 8.8368 & -29.9433 \\
\hline 50 & 8.887 & 0.3321 \\
\hline 51 & 8.9236 & -0.6415 \\
\hline 52 & 8.9419 & 24.9826 \\
\hline 53 & 8.9589 & 12.3643 \\
\hline 54 & 8.9949 & -78.325 \\
\hline 55 & 9.0366 & 17.7006 \\
\hline 56 & 9.085 & 25.7615 \\
\hline 57 & 9.1037 & 43.4152 \\
\hline 58 & 9.1117 & -14.8319 \\
\hline 59 & 9.1652 & 11.9091 \\
\hline 60 & 9.1816 & -5.4217 \\
\hline
\end{tabular}

* $\mathrm{R}$ (velocity) $10^{* *}$-40 erg-esu-cm 
Table S9. Calculated specific OR data for (3S)-1.

\begin{tabular}{|l|l|}
\hline Conf & Alpha[D](vacuum) \\
\hline C1 & -154.82 \\
\hline Experimental Value & -242.00 \\
\hline
\end{tabular}




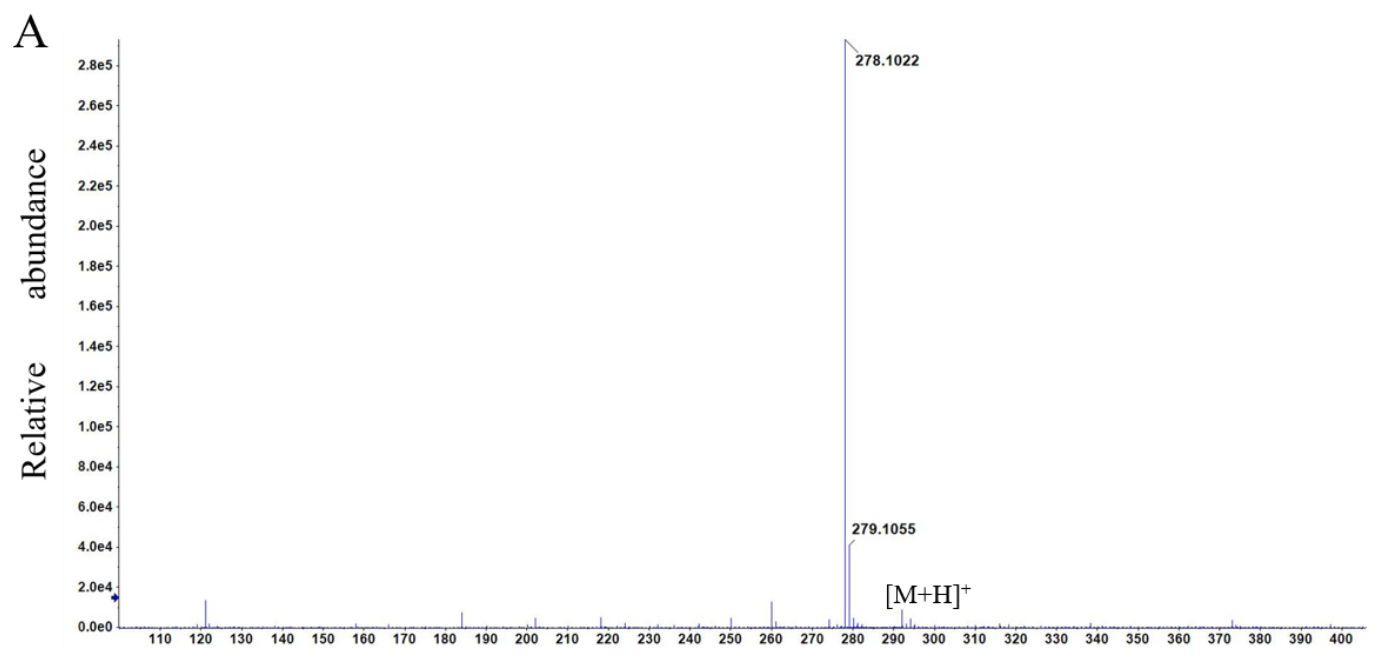

B
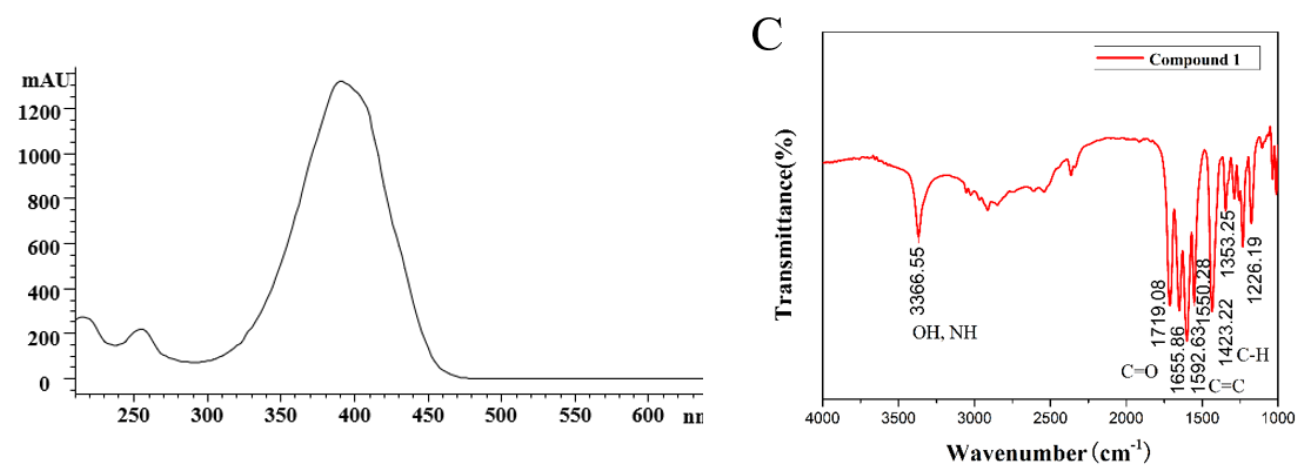

Figure S1. Determination of the structure of compound 1 .

(A) HRMS spectrum of compound 1. (B)UV-visible absorption of compound 1. (C) Infrared spectrum of compound $\mathbf{1}$. 


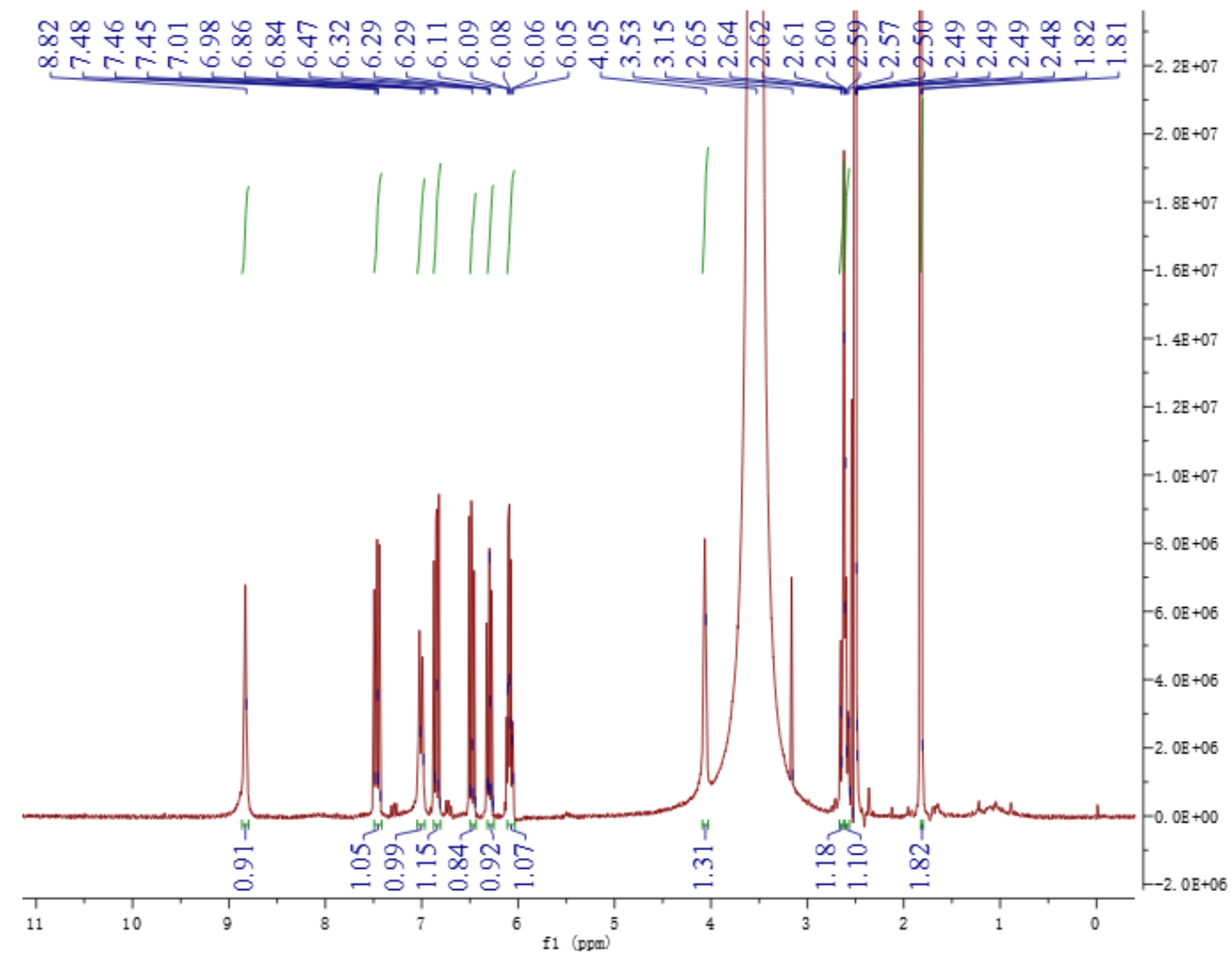

Figure S2. ${ }^{1} \mathrm{H}$ NMR spectrum of $\mathbf{1}$ in DMSO (500 MHz) 


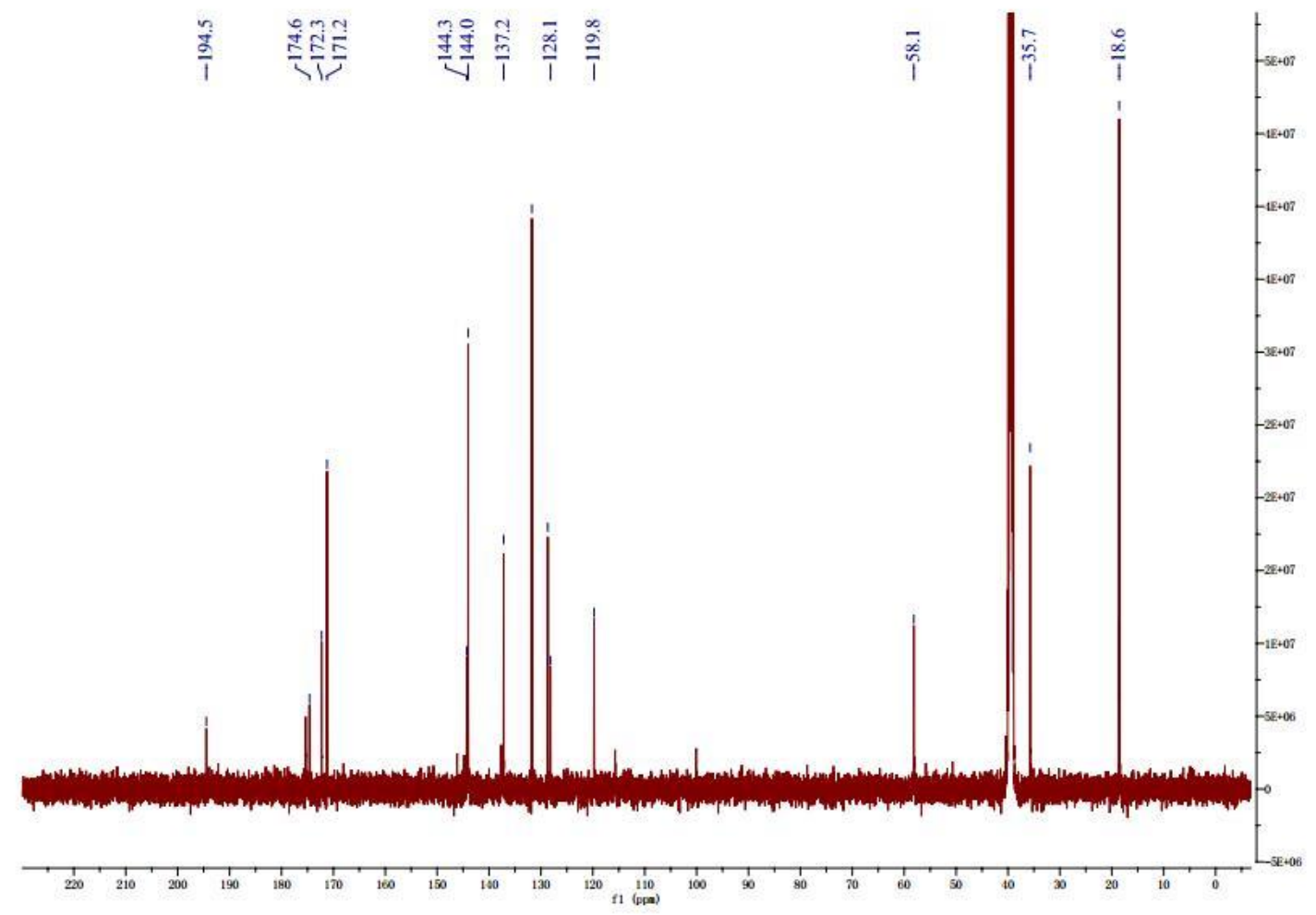

Figure S3. ${ }^{13} \mathrm{C}$ NMR spectrum of $\mathbf{1}$ in DMSO 


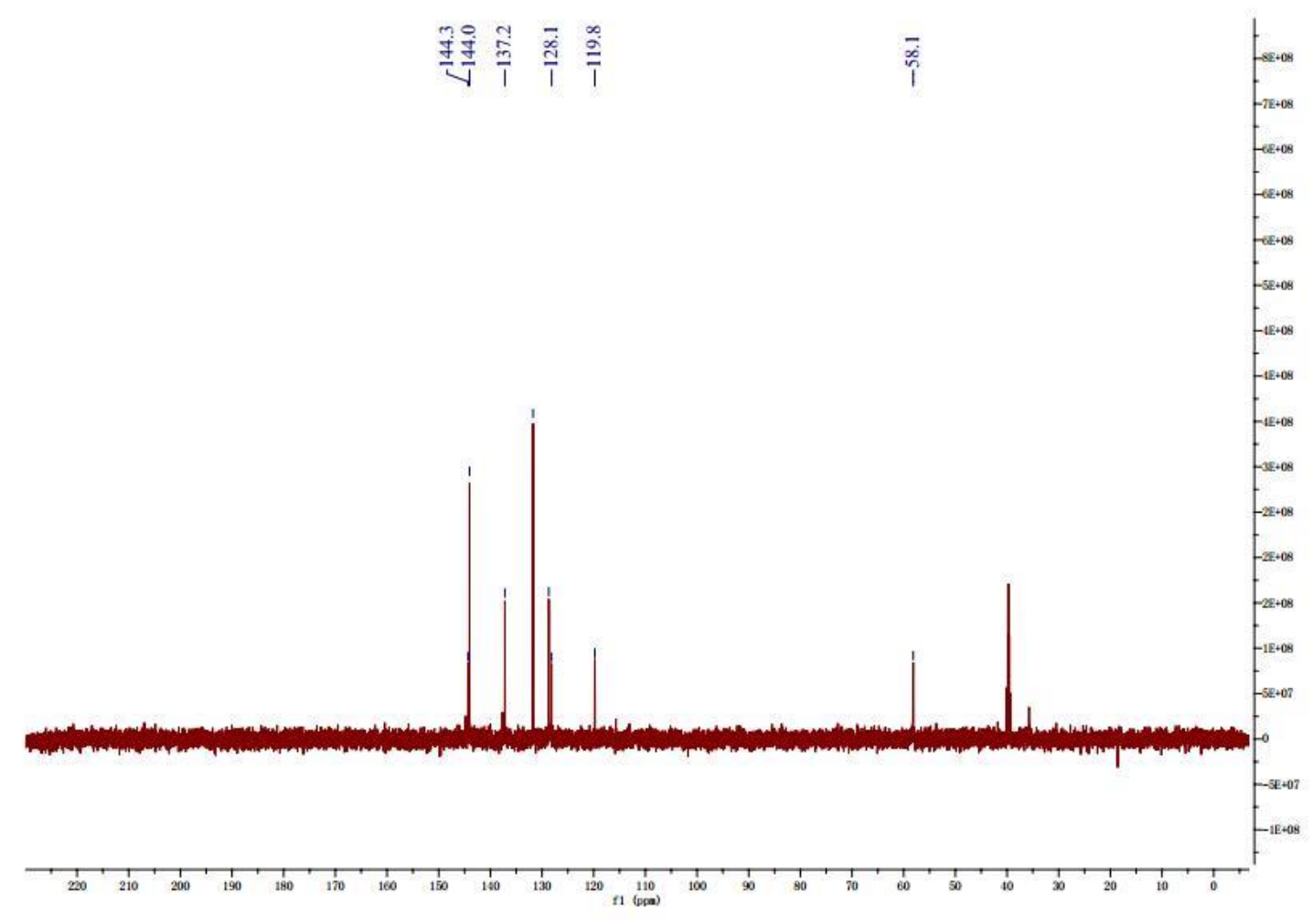

Figure S4. DEPT 90 NMR spectrum of 1 in DMSO 


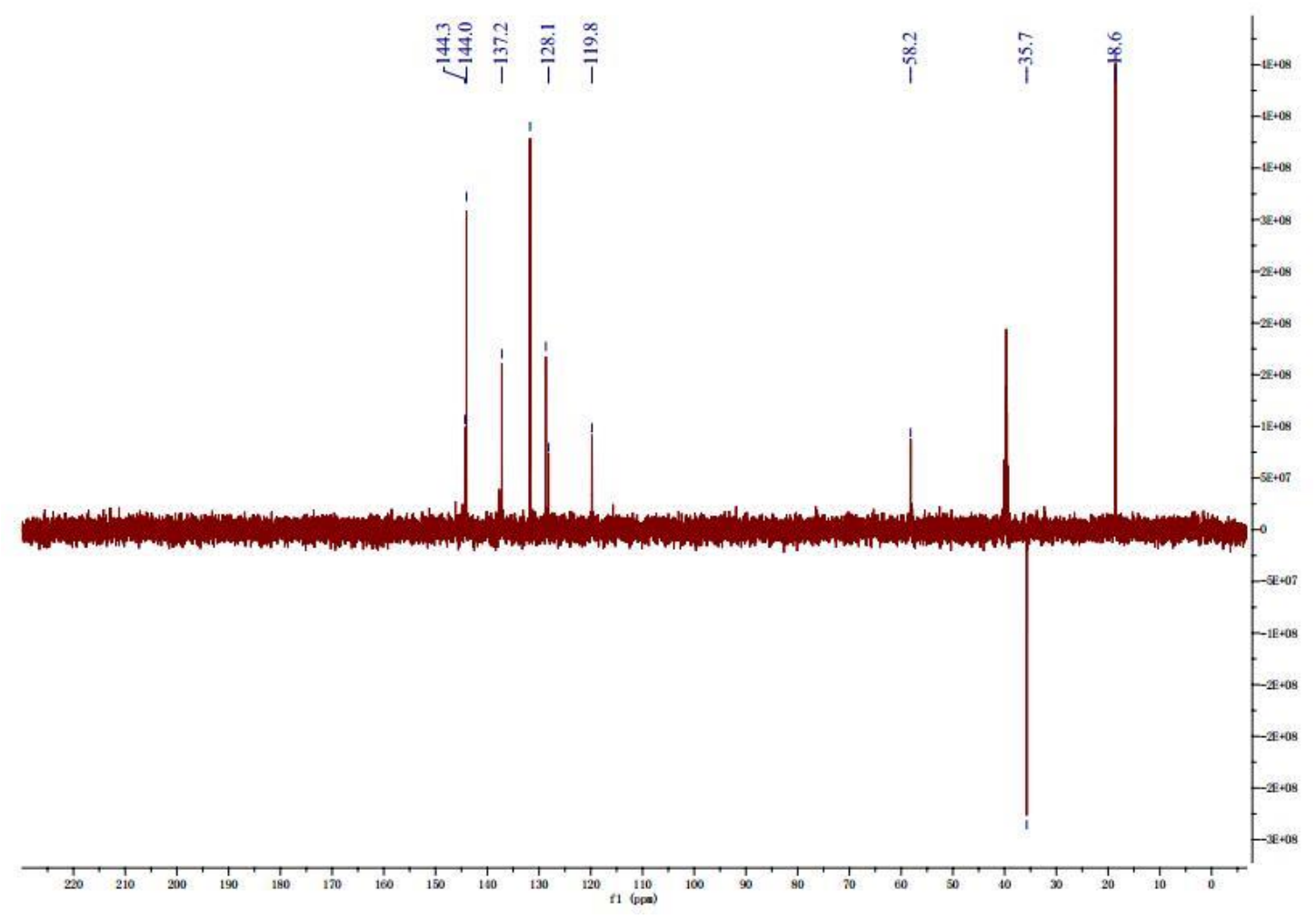

Figure S5. DEPT 135 NMR spectrum of 1 in DMSO 


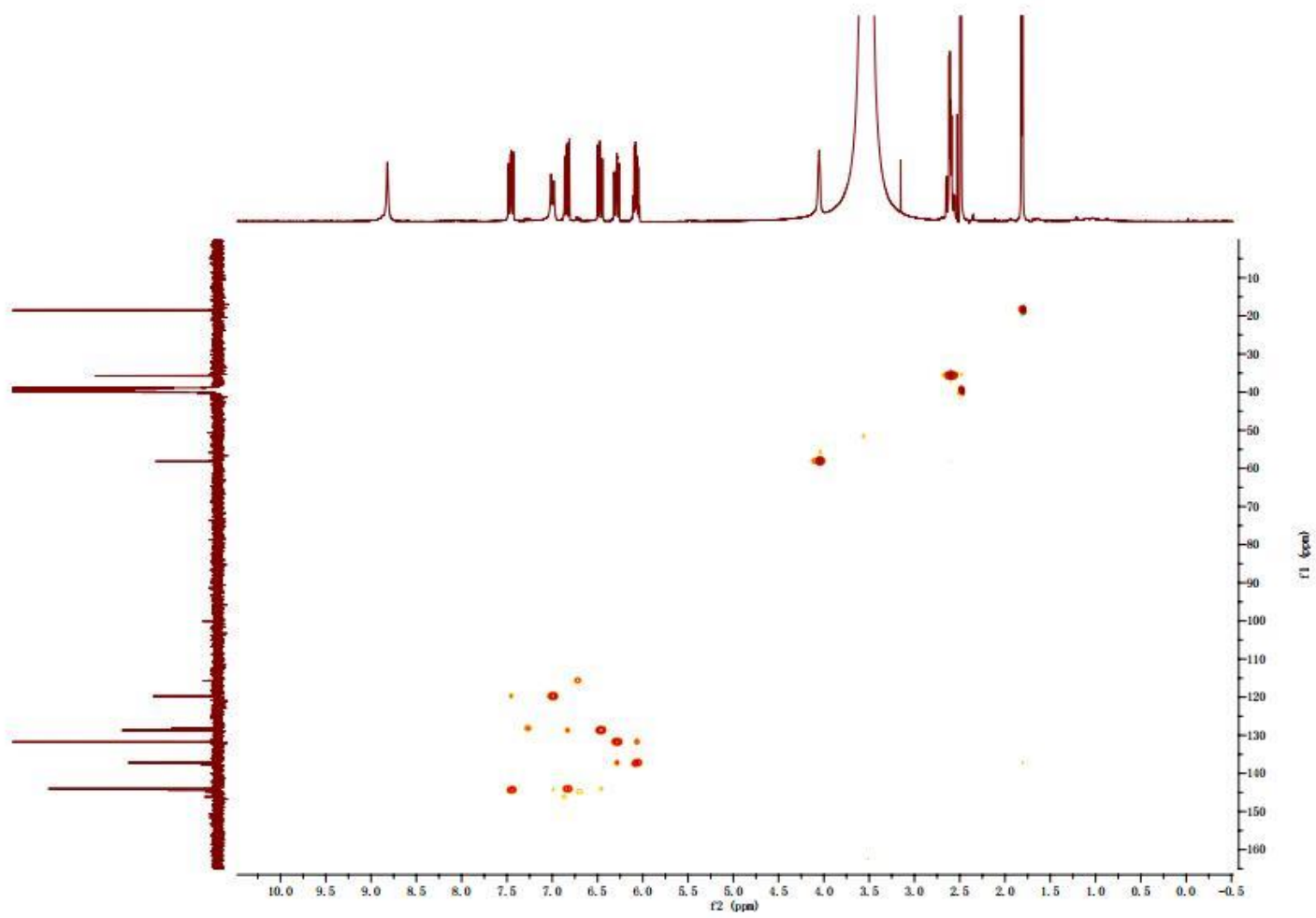

Figure S6. The HSQC spectrum of 1 in DMSO 


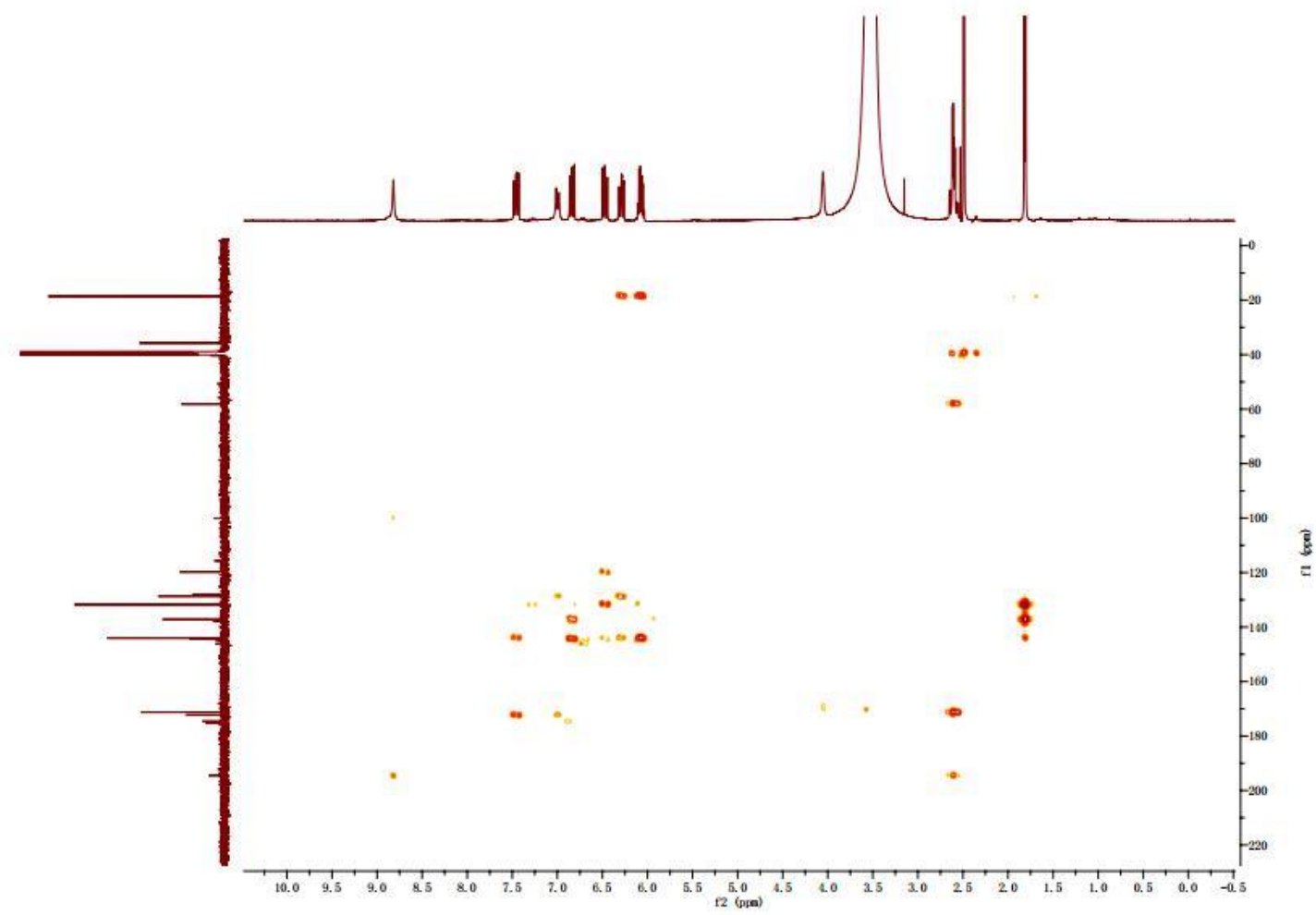

Figure S7. The HMBC spectrum of $\mathbf{1}$ in DMSO 

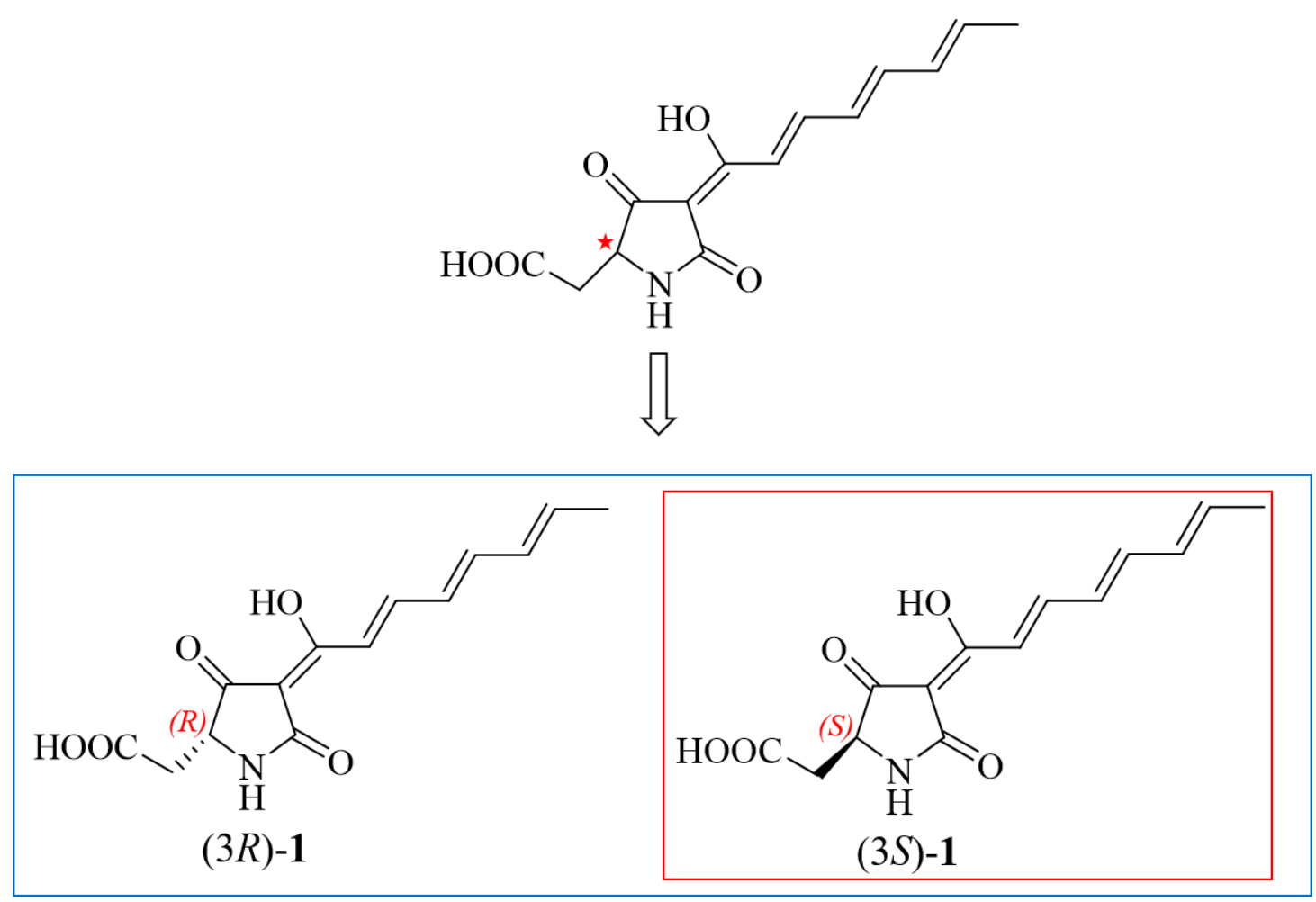

Figure S8. Two candidate absolute configurations of $\mathbf{1}$ 


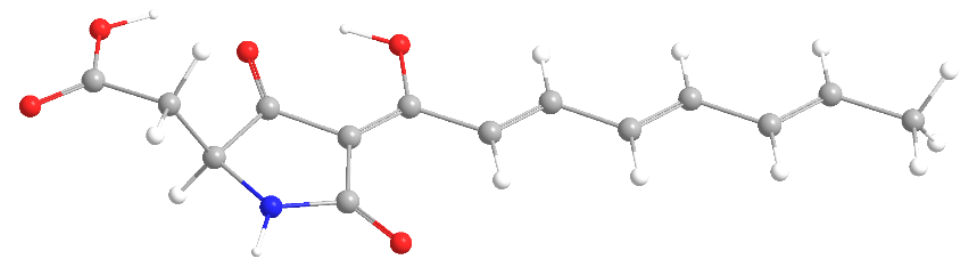

Figure S9. B3LYP/6-31G(d) optimized lowest energy conformer for (3S)-1 
A05163-DKC PI HFI STS GVLTREEVQADGVASS. AAANE. P. . PADGSEGYI AS KWASERLLER CPAA-DKC PI HFVS TS A. . . . . . SDPS AT PSS. AI S YS. P. . PLDGS DGYVS S KUVSERI LER FsdS-DKC PI HFI SS GS VR. . . I YGDEECPKI YNI DQS LPI S PTPPSDGYVAS KWAAEKFLRT

ExqS-DKC PI HFLSSGAVI . . KYNS GLAP. . . . . . . . PADGGDGYVATKWASEAFLRQ

TtcA-DKC PI HFVSTS AVL. . . . SDPLAS PSS. ANS YS. P. . PPDGSDGYI AS KWASERLLER $\star \star \star \star$

Figure S10. Multiple alignment of DKC domain sequences 


\section{References}

1. Cao, F.; Cheng, J. T.; Chen, X. A.; Li, Y. Q.; Mao, X. M. Development of an efficient genetic system in a gene cluster-rich endophytic fungus Calcarisporium arbuscula NRRL 3705. J. Microbiol. Methods. 2018, 151, 1-6. 